Rev. Elev. Méd. vét. Pays trop., 1979, 32 (3) : 233-239.

\title{
La maladie nodulaire cutanée des bovins II. - Production d'un vaccin lyophilisé à virus vivant
}

\author{
par P. C. LEFEVRE (*)
}

(avec la collaboration technique de Z. GNALDAM et R. KOSKAL)

\begin{abstract}
RÉSUMÉ
Un vaccin lyophilisé à virus vıvant a été produit contre la maladie nodulaire cutanée des bovins. Dans cet article, la technique de sa production, la stabilité du produit lyophılisé et celle du prodult reconstitué sont étudiées. La lyophılisation n'entraîne aucune baisse de titre quel que soit le milieu de lyophilisatıon utilisé. Par contre, le milieu à la néopeptone assure une me1lleure thermoprotection du produit lyophilssé. Une fois reconstitué, ce vaccin est relativement stable à la chaleur, mass doit être protégé de la lumière solaire. Il donne, alors, satisfaction dans la prévention de la maladie en brousse.
\end{abstract}

\section{INTRODUCTION}

Depuis une dizaine d'années la maladie nodulaire cutanée des bovins (MNCB) ou lumpy-skin disease des anglo-saxons est apparue dans de nombreux pays du centre et de l'ouest africain (11-1-6-7).

L'extension de la maladie et sa pérennité dans les pays déjà atteints ne peuvent que se poursuivre si aucune mesure prophylactique n'est prise.

Dans le but de prévenir l'apparition de la maladie dans les pays encore indemnes et de limiter le nombre de foyers dans les pays contaminés, un vaccin a été mis au point au Laboratoire de Farcha.

Le présent article fait la synthèse des travaux menés depuis 2 ans sur :

- la courbe de croissance du virus-vaccin,

- la production du vaccin,

- la stabilité du produit lyophilisé,

- la stabilité du produit reconstitué.

(*) Institut d'Elevage et de Médecine Vétérinaire des Pays Tropicaux. Laboratoire de Farcha, B. P. 433, N'Djaména, Républıque du Tchad.
La détermination de la dose minimale vaccinale ainsi que la réponse immunitaire après vaccination seront étudiées dans un prochain article.

Il est possible, dès à présent, d'affirmer que ce vaccin utilisé en brousse à la dose recommandée par WEISS de 12000 DICT 50 par animal donne entière satisfaction.

\section{MATÉRIEL ET MÉTHODES}

\section{A) Souche}

Virus de la maladie nodulaire isolé à Madagascar (10) et atténué par 101 passages sur cellules de rein de lapin. La "banque » de virus d'ensemencement est obtenue par 5 passages sur néphrocytes embryonnaires de veau.

\section{B) Courbe de croissance}

Des boîtes de Roux avec tapis cellulaire complet sont inoculées et incubées à $37^{\circ} \mathrm{C}$. Toutes les 24 h, 2 boîtes sont retirées de l'étuve: la première est congelée immédiatement après 
raclage du tapis cellulaire; seul le milieu de la deuxième est récolté et congelé. Après 3 cycles de congélation-décongélation, des titrages sont effectués sur le milieu seul et sur le milieu plus cellules. Cette expérience est recommencée plusieurs fois en faisant varier le taux d'infectivité.

\section{C) Production du vaccin}

La banque du virus-vaccin est inoculée à des néphrocytes embryonnaires de veau au $2^{\mathfrak{e}}$ passage, entretenus dans un milieu sans anticorps anti-MNCB (origine européenne). La récolte se fait quand l'effet cytopathique est complet et après 3 cycles de congélation-décongélation.

La suspension virulente obtenue est mélangée à parties égales avec le milieu de lyophilisation. Le mélange est réparti sous froid à raison de $5 \mathrm{ml}$ par flacon puis porté dans le lyophilisateur: lyophilisation primaire de $-60^{\circ} \mathrm{C}$ à $0^{\circ} \mathrm{C}$ en $16 \mathrm{~h}$ puis lyophilisation secondaire de 0 à $37^{\circ} \mathrm{C}$ en $18 \mathrm{~h}$ supplémentaires. Les flacons sont bouchés sous atmosphère d'azote et conservés à $-20^{\circ} \mathrm{C}$.

Les milieux de lyophilisation employés sont : lot $\mathrm{A}=$ milieu dit de WEYBRIDGE (2) lot $\mathrm{B}=$ milieu de WIKTOR (8) lot $\mathrm{C}=$ solution de néopeptone à 11 p. 100 .

\section{D) Stabilité du produit lyophilisé}

Des flacons de chacun des 3 lots sont placés à $-20^{\circ},+4^{\circ}$ et $+37^{\circ} \mathrm{C}$. Au bout de $3,6,9$ et 12 mois, 5 flacons de chaque lot et à chaque température sont prélevés et titrés.

\section{E) Stabilité du produit reconstitué}

1) Effet de la température : Des flacons de chaque lot sont reconstitués dans $100 \mathrm{ml}$ de sérum physiologique ou d'une solution molaire de sulfate de magnésium, préalablement portés à $37^{\circ} \mathrm{C}$. Les titrages ont lieu immédiatement après reconstitution puis après $1,2,3$ et $4 \mathrm{~h}$ à l'étuve.

2) Dans les conditions d'utilisation en brousse (effet de la lumière). Deux flacons de chaque lot sont reconstitués dans $100 \mathrm{ml}$ de sérum physiologique froid $\left(+4^{\circ} \mathrm{C}\right)$. Un flacon est enveloppé d'un linge humide, l'autre non. Les deux flacons sont alors exposés sur le rebord d'une fenêtre et les titrages ont lieu comme précédemment.

\section{F) Titrages}

Les titrages sont effectués en tubes de cellules embryonnaires de rein de veau : 5 tubes par dilution ; lecture au $14^{\mathrm{e}}$ jour.

\section{RÉSULTATS}

\section{A) Courbe de croissance}

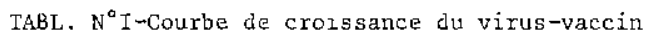

\begin{tabular}{|c|c|c|c|c|}
\hline \multirow{2}{*}{$\begin{array}{l}\text { Temps } \\
\text { en } \\
\text { heures }\end{array}$} & \multicolumn{2}{|c|}{$\begin{array}{r}\text { Rapport virus/ } \\
\text { cellules (1p.100) }\end{array}$} & \multicolumn{2}{|c|}{$\begin{array}{l}\text { Rapport virus/ } \\
\text { cellules ( } 20 \text { p.100) }\end{array}$} \\
\hline & $\begin{array}{c}\text { Milieu } \\
+\quad \text { cellules }\end{array}$ & Milıeu & $\begin{array}{c}\text { Mulieu } \\
+ \text { cellules }\end{array}$ & Milieu \\
\hline 24 & $\mathrm{~N} \mathrm{~T}$ & $\mathrm{~N} \mathrm{~T}$ & $3.2 \pi$ & 2.2 \\
\hline 48 & 2.6 & 1.8 & 4.2 & 2.2 \\
\hline 72 & 5.2 & 3.8 & 6.8 & 4.2 \\
\hline 96 & 5.2 & 4.6 & 6.6 & 5.2 \\
\hline 120 & 5.6 & 5.6 & 5.6 & 4.6 \\
\hline $144(6 j)$ & 5.8 & 5.8 & 5.8 & 5.8 \\
\hline $168(7 \mathrm{j})$ & 6.2 & 4.8 & 7 & 6.2 \\
\hline $192(8 \mathrm{~J})$ & 6.2 & 5 & $\mathrm{~N} \mathrm{~T}$ & $N \mathrm{~J}^{\prime}$ \\
\hline 2$] 6(9 \mathrm{j})$ & 5.8 & 5.2 & $\mathrm{~V} \mathrm{~T}$ & $N \mathrm{~T}$ \\
\hline
\end{tabular}

N $\mathrm{T}=$ non tilrê; log decimal de la DICTSO/mI

Il apparaît que le titre maximal est atteint en 7 jours avec un inoculum riche et en 9 jours avec un faible inoculum. Pour l'obtention d'un titre élevé, il est nécessaire de récolter la totalité du tapis cellulaire. Cette observation s'accorde avec celle de PLOWRIGHT et WITCOMB (11) qui constatent que le virus reste lié aux cellules.

\section{B) Effet de la lyophilisation sur le titre du vaccin}

TABL, N ${ }^{\circ}$ II-TItre avant et après lyophilisation

\begin{tabular}{|c|c|c|c|}
\hline \multirow{2}{*}{ Lot } & $\begin{array}{c}\text { Avant } \\
\text { 1yophilisation }\end{array}$ & \multicolumn{2}{|c|}{ Après lyophilisation } \\
\cline { 2 - 4 } & Sérum physiol. Sol. Mg SO 4 \\
\hline A & $4.8^{*}$ & 4.5 & 4.9 \\
B & 4.6 & 4.1 & 4.9 \\
C & 4.6 & 4.7 & 4.5 \\
\hline
\end{tabular}

$\because$ Log decimal de la DICT 5p/ml.

La lyophilisation n'entraîne pas de baisse du titre et les 3 milieux ont le même effet protecteur.

\section{C) Stabilité du produit lyophilisé}

Les graphiques $\mathrm{A}, \mathrm{B}$ et $\mathrm{C}$ donnent, pour chacun des lots, l'évolution du titre du vacccin conservé à $-20^{\circ} \mathrm{C},+4^{\circ} \mathrm{C}$ et $\times 37^{\circ} \mathrm{C}$. 
Les courbes sont de la forme :

$$
\begin{aligned}
y & =a \cdot e^{x \log \mathrm{k}} \\
\operatorname{lot} \mathrm{A}-20^{\circ} \mathrm{C}: \mathrm{y} & =3,71 \mathrm{e}^{-0,009 t} \\
+4^{\circ} \mathrm{C}: \mathrm{y} & =3,74 \mathrm{e}^{-0,01 \mathrm{t}} \\
+37^{\circ} \mathrm{C}: \mathrm{y} & =3,73 \mathrm{e}^{-0,28 t} \\
\text { lot } \mathrm{B}-20^{\circ} \mathrm{C}: \mathrm{y} & =3,64 \mathrm{e}^{-0,0064 t} \\
+4^{\circ} \mathrm{C}: \mathrm{y} & =3,49 \mathrm{e}^{-0,0043 t} \\
+37^{\circ} \mathrm{C}: \mathrm{y} & =3,60 \mathrm{e}^{-0,21 \mathrm{t}} \\
\text { lot C-20 }-20^{\circ} \mathrm{C}: \mathrm{y} & =3,30 \mathrm{e}^{-6,002 t} \\
+4^{\circ} \mathrm{C}: \mathrm{y} & =3,44 \mathrm{e}^{-0,007 t} \\
+37^{\circ} \mathrm{C}: \mathrm{y} & =2,88 \mathrm{e}^{-0,07 t}
\end{aligned}
$$

Ces graphiques montrent bien la bonne stabilité des produits lyophilisés et conservés à $-20^{\circ} \mathrm{C}$ et $+4{ }^{\circ} \mathrm{C}$ et, au contraire, la chute marquée du titre à $+37^{\circ} \mathrm{C}$.

Toutefois, il semble que le milieu à la néopeptone assure une meilleure protection que les 2 autres (exposant de e : 0,07 contre 0,21 et $0,28)$.

Une hypothèse pouvant expliquer ce phénomène serait la très faible humidité résiduelle obtenue grâce à l'absence de saccharose dans le milieu à la néopeptone.

GRAPHIQUE A : Conservation du lot $\mathrm{A}$ a $-20^{\circ} \mathrm{C},+4^{\circ} \mathrm{C}$ et $37^{\circ} \mathrm{C}$

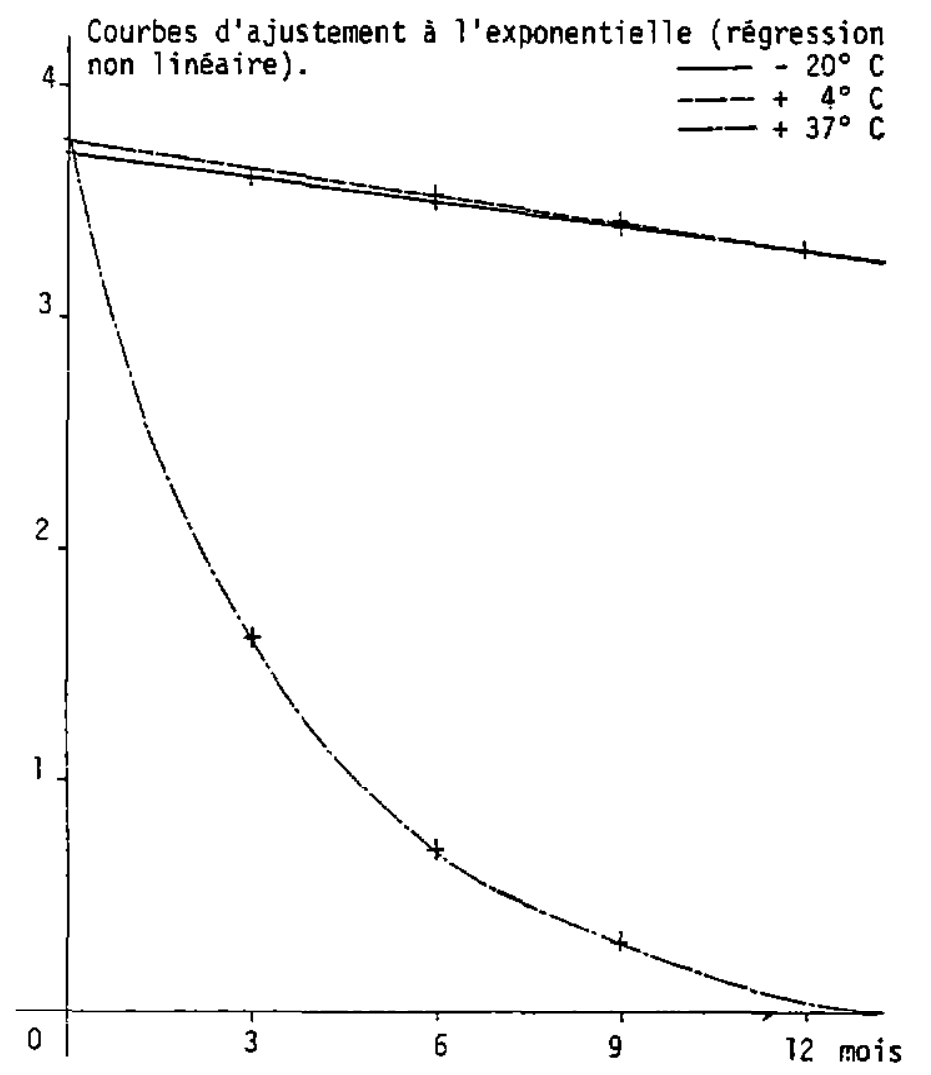


GRAPHIQUE B : Conservation du $\operatorname{lot} \mathrm{B}^{\circ} \mathrm{a}-20^{\circ} \mathrm{C},+4^{\circ} \mathrm{C}$ et $+37^{\circ} \mathrm{C}$

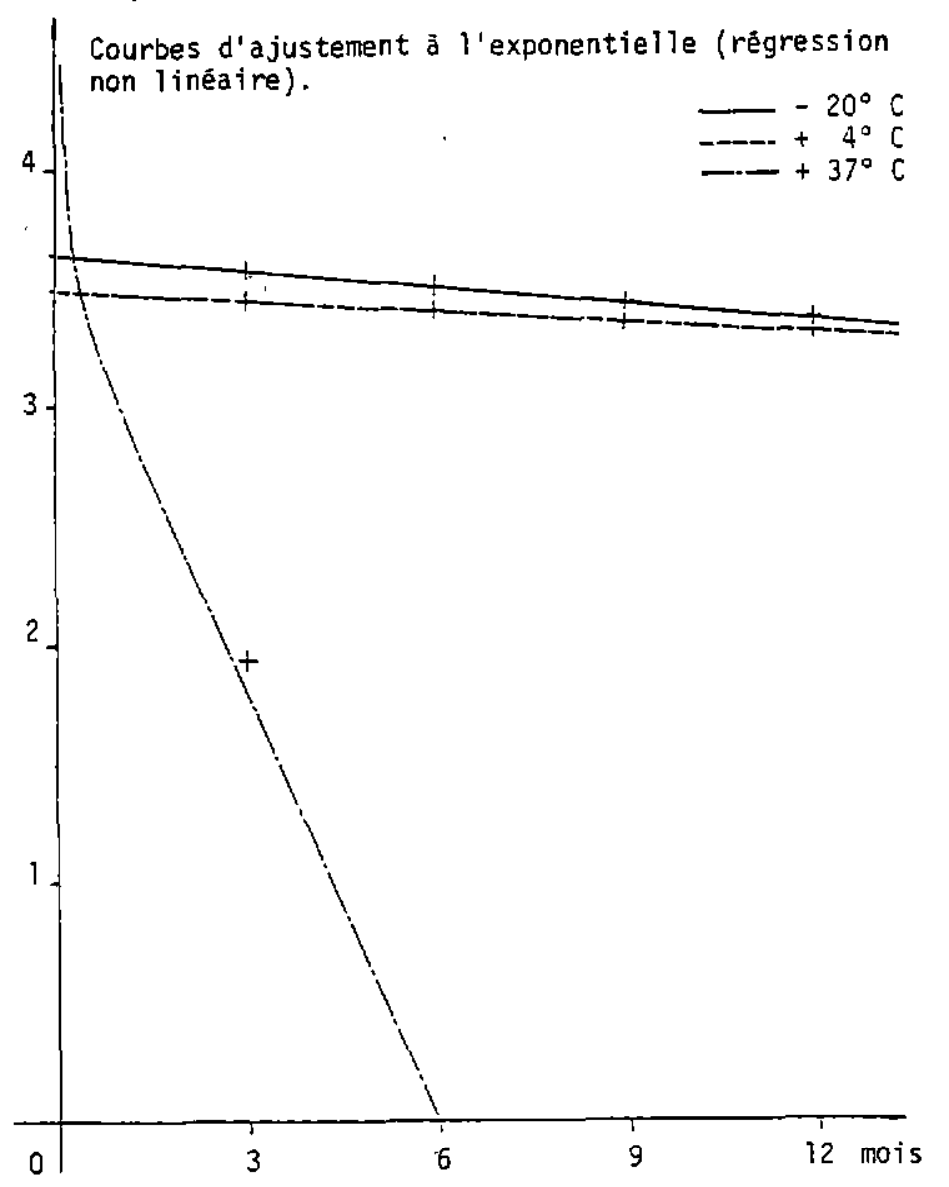

GRAPHIQUE $\mathrm{C}$ : Conservation du lot $\mathrm{C}$ a $-20^{\circ} \mathrm{C}, 44^{\circ} \mathrm{C}$ et $37^{\circ} \mathrm{C}$

4 Courbes d'ajustement à l'exponentielle (régression non 1 inéajre).

$-20^{\circ} \mathrm{C}$

$--+4^{\circ} \mathrm{C}$

$-37^{\circ} \mathrm{C}$

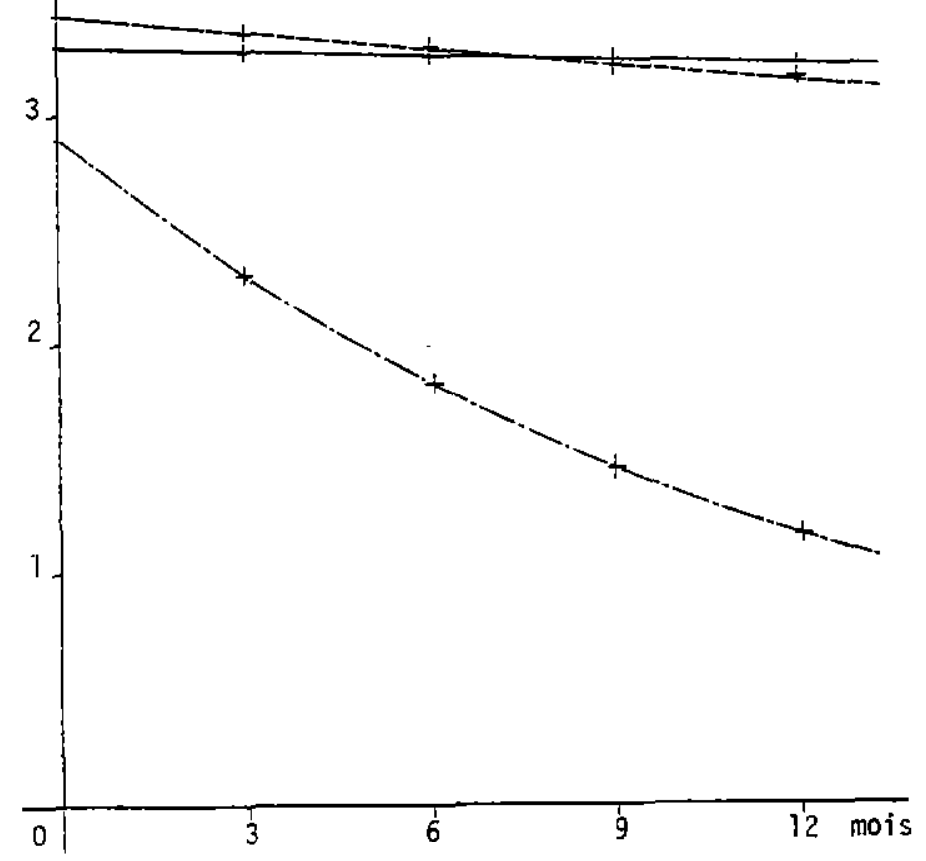


D) Stabilité du produit reconstitué

a) Effet de la chaleur

\begin{tabular}{|c|c|c|c|c|c|c|}
\hline & \multicolumn{3}{|c|}{$\begin{array}{l}\text { Dans sérum } \\
\text { physiologique }\end{array}$} & \multicolumn{3}{|c|}{$\begin{array}{l}\text { Dans solution } \\
\mathrm{Hs} \mathrm{SO}_{4}, 1 \mathrm{M}\end{array}$} \\
\hline & Lot $A$ & Lot B & Lot $\mathrm{C}$ & Lot $A$ & Lot B & Lot C \\
\hline 0 & $3.8 \%$ & 3.6 & 3.8 & 3.6 & 3.6 & 3.4 \\
\hline $1 / 2 \mathrm{H}$ & 3.7 & 3.7 & 3.8 & 3.5 & 3.7 & 3.4 \\
\hline $1 \mathrm{H}$ & 3.5 & 3.6 & 3.4 & 3.2 & 3.5 & 3.3 \\
\hline $2 \mathrm{H}$ & 3.2 & 3.6 & 3.3 & 3.2 & 3.5 & 3.4 \\
\hline $3 \mathrm{H}$ & 3.1 & 3.3 & 3.1 & 2.8 & 3 & 3 \\
\hline $4 \mathrm{H}$ & $\mathrm{NT}$ & NT & 3.2 & $\mathrm{~N} T$ & $\mathrm{~N} \mathrm{~T}$ & 3.2 \\
\hline
\end{tabular}

$\because$ Log. décimal de la DICT $50 / \mathrm{ml}$ - moyenne de 3 titrages.

$\mathrm{N} \mathrm{T}=$ non titrë. b) Dans les conditions de brousse

Les tableaux et le graphique sont très éloquents: la chaleur n'entraîne qu'une baisse relativement faible par rapport à celle due à l'exposition à la lumière. Dans les conditions de brousse, le vaccin doit donc être efficacement protégé contre la lumière solaire.

GRAPHIQUE $D$ : Conservation des lots $A, B, C$ après reconstitution

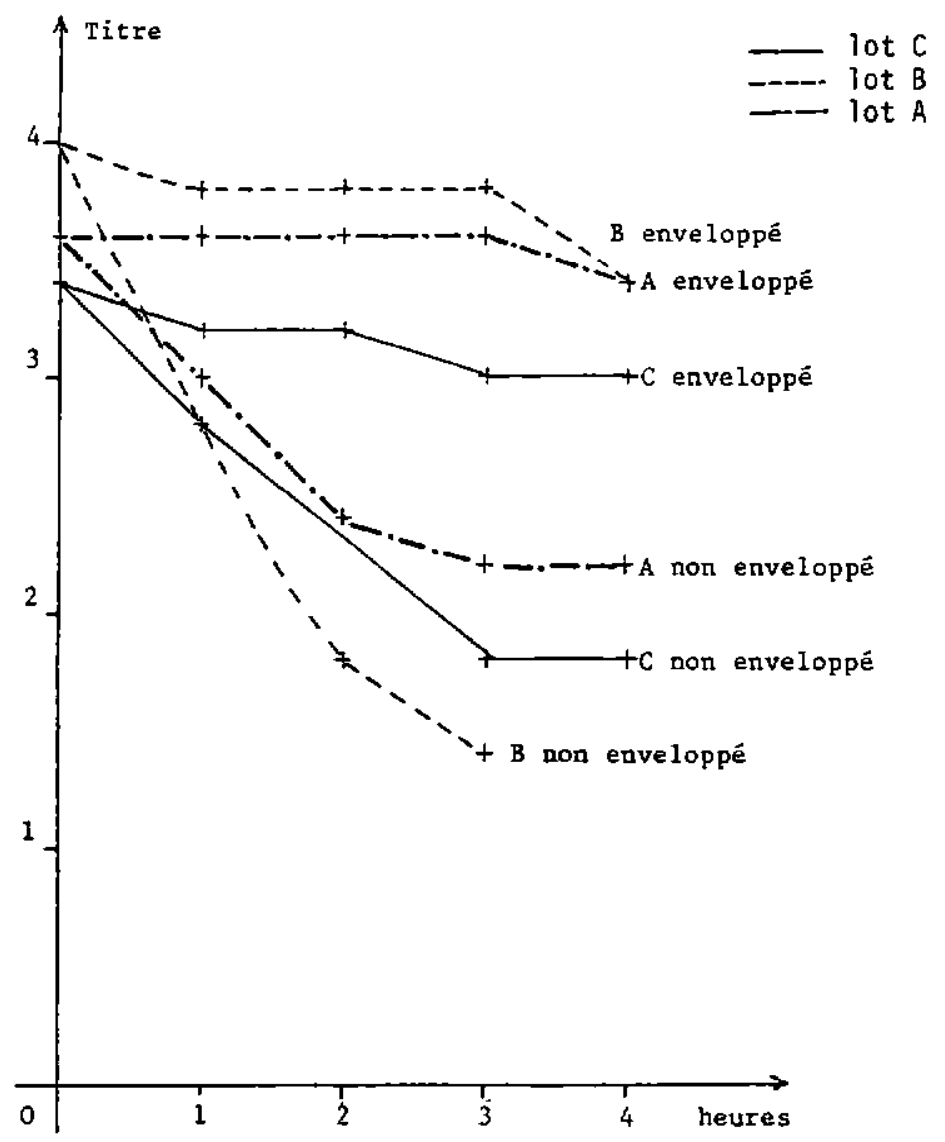




\section{CONCLUSIONS}

La lutte contre la MNCB passe obligatoirement par la vaccination en zone contaminée. Le vaccin à virus vivant est facile à produire et se comporte relativement bien une fois lyophilisé.

Les principaux points de la production du vaccin sont :

- La nécessité de récolter la totalité du milieu et des cellules infectées et de libérer le maximum de virus par 3 cycles de congélationdécongélation ;

-_ La lyophilisation n'entraîne aucune baisse de titre quelque soit le milieu protecteur utilisé ;
- Par contre, le milieu de lyophilisation joule un rôle important dans la thermoprotection du vaccin lyophilisé. Le milieu à la néopeptone à 11 p. 100 assure une bonne conservation du produit ;

- Lors de la reconstitution, l'utilisation d'une solution molaire de sulfate de magnésium ne semble pas prolonger la survie du virus ;

- Dans les conditions d'utilisation en brousse, le vaccin doit être soigneusement protégé de la chaleur et surtout de la lumière. Cette protection apportée par un linge mouillé est, du reste, classique dans les pays du sahel depuis les campagnes de vaccination anti-bovipestique.

\section{SUMMARY}

Lumpy skin disease. II. - Production of a freeze dried vaccine

A freeze dried attenuated virus vaccine is produced to protect cattle against lumpy skin disease. The technics of production, the stability of the freeze dried product and the stability of the reconstituted vaccine are studied.

No loss of titer is seen of ten freeze drying whatever the protective medium used. The medium with neopepton (11 p. 100) assures a better protection of the freeze dried vaccine against heat.

Once reconstituted the vaccine is relatively thermostable but must be protected against sun light.

\section{RESUMEN}

\section{La dermatosis nodular de los bovinos. II. - Producción de una vacuna} liofilizada

Una vacuna liofilizada a virus vivo fué producido contra la dermatosis nodular de los bovinos.

Se estudian sucesivamente la técnica de su producción, la estabilidad del producto liofilizado y la del producto reconstituido. La liofilización no ocasiona ninguna disminución del título cualquiera que sea el medio de liofilización utilizado. En cambio, el medio a la neopeptona asegura una mejora termoprotección del producto liofilizado.

En cuanto reconstituida, dicha vacuna es relativamente estable para con el calor, pero necesita una protección contra la luz del sol. Entonces da satisfacción para la prevención de la enfermedad en sabana con matorrales.

\section{BIBLIOGRAPHIE}

1. BOURDIN (P.). La maladie nodulaire cutanée des bovins. Paris, Expansion scientifique française, 1970,100 p. (Coll. les Maladies à virus).

2. BOYCE (K. J.), EDGAR (A. W.). Production of freeze Bricella abortus strain 19 vaccine. $J$. appl. Bact., 1966, 29 : 401-408.

3. CAPSTICK (J. B.), COACKLEY (W.). Protection against lumpsy skin disease. 1) Trials with a vaccine against Neethling type infection. Res. vet. Sci., 1961, 2 (4) : 362-368.

4. CAPSTICK (P. B.), COACKLEY (W.). Protection of cattle against lumpy skin disease. II) Factors affecting small scale production of a tissue culture propagated virus vaccine. Rev. vet. Sci., 1961, 2 (4) : 369-374.
5. CAPSTICK (P. B.), COACKLEY (W.). Lumpy skin disease. The determination of the immune state of cattle by intradermal test. Res. vet. Sci., 1962, 3 : 287-291.

6. LEFEVRE (P. C.), BONNET (J. B.), VALLAT (B.). La maladie nodulaire cutanée des bovins. I. - Situation épizootiologique actuelle en Afrique. Rev. Elev. Méd. vét. Pays trop., 1979, 32.

7. NAWATHE (D. R.), GIBBS (E. P. J.), ASAGBA (M. O.), LAW MAN (M. J. P.). Lumpy skin disease in Nigeria. Trop. anim. Hlth. Prod., 1978, $10: 49$ 54.

8. OMS. La rage. Techniques de laboratoire. Genève, OMS, 1967. n 23. 
9. PLOWRIGHT (W.), WHITCOMB (M. A.). The growth in tissue culture of a virus derived from lumpsy skin disease of cattle. J. Path. Bact., 1959, 78 : 397-406.

10. RAMISSE (J.), SERRES (H.), RAKOTONDRAMARY (E.). Isolement à Madagascar de virus asso- ciés à la dermatose nodulaire bovine. Rev. Elev. Méd. vét. Pays trop., 1969, 22 (3) : 357-362.

11. Rapports annuels du laboratoire de Farcha. I. E. M. V. T., 1976-1977-1978.

12. WEISS (K. E.), Dermatose nodulaire in: Maladies nouvelles des animaux. Rome, F. A. O., 1964. (Co1l. Etudes agricoles F. A. O. $n^{\circ}$ 61.) 